\title{
EFEITOS DE EXTRATOS DE Nierembergia veitchii (Hook) Solanaceae SOBRE A FERTILIDADE DE RATAS E MORFOLOGIA ÓSSEA DOS FETOS
}

\author{
THE EFFECTS OF Nierembergia veitchii (Hook) Solanaceae EXTRACTS IN \\ RAT FERTILITY AND FETAL SKELETON MORPHOLOGY
}

\author{
João Roberto Braga de Mello ${ }^{1}$ Augusto Langeloh ${ }^{1}$ Gerhard Habermehl $^{2}$
}

Hans Krebs $^{2}$ Fernanda Bastos $^{3}$ Fernanda Alvares $^{4}$

\section{RESUMO}

\begin{abstract}
Os efeitos da administração oral dos extratos aquo so $\left(\mathrm{NvH}_{2} \mathrm{O}\right)$, metanólico (NvmeOH), butanólico (Nvbut) e hexano (Nvhex), obtidos, seqüencialmente, de $500 \mathrm{~g}$ de Nierembergia veitchii (planta seca), foram comparados aos obtidos com vitamina $D_{3}\left(2,5 \mathrm{mg}_{\mathrm{kg}} \mathrm{kg}^{-1}\right.$ ) e aos de um grupo controle (solução fisiológica - SF), quando administrados, durante todo o período de gestação (21 dias), a ratas albinas Wistar. A redução do ganho de peso foi evidente nas fêmeas tratadas com Nvhex, Nvbut e vitamina $D_{3}$. Nesses grupos, houve redução do número de implantes uterinos $(9,3+1,4 ; 9,6+1,4$ e 8,4 + 1,4), quando comparados com o controle $(S F)(1 \overline{1,2} \pm 0,4)$. Essa redução também foi observada no número de filhotes por ninhada $(8,1+1,3 ; 9,0$ $+1,5 ; 5,1+1,7$ e 10,3 +0,6), e na massa corporal dos filhotes

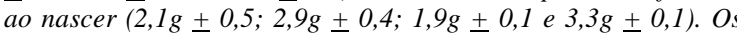
resultados obtidos com $\mathrm{NvH}_{2} \mathrm{O}$ e $\mathrm{NvmeOH}$ não diferiram dos obtidos com o grupo controle. Anomalias macroscópicas foram observadas em fetos de ratas tratadas com Nvhex e Nvbut, 0,1 $0,2 \%$ respectivamente. Os resultados da análise da morfologia óssea dos fetos mostraram a ocorrência de 33,3\%; 45\%; 9,8\% $11,1 \%$ de anomalias para os grupos $\mathrm{NvH}_{2} \mathrm{O}$, NvmeOH, Nvbut e $S F$, respectivamente. As anomalias ósseas mais freqüentemente observadas ocorreram no crânio e constaram de ossificação incompleta de supraoccipital, interparietais, parietais, frontal e temporal.
\end{abstract}

Palavras-chave: plantas calcinogênicas, Nierembergia veitchii, vitamina $D_{3}$, infertilidade, teratogenia.

\section{SUMMARY}

The effects of oral administration of aqueous $\left(\mathrm{NvH}_{2} \mathrm{O}\right)$, methanolic (NvmeOH), buthanolic (Nvbut) and hexan (Nvhex) extracts, sequentialy obtained from $500 \mathrm{~g}$ Nierembergia veitchii (dried plant) were compared with a vitamin $D_{3}$ treated group and with a control group (saline), when administered during the pregnancy to Wistar rats (21 days). Weigth gain was reduced in dams treated with Nvhex, Nvbut and vitamin $D_{3}$. In these groups it was observed a reduction in uterine implantations $(9.3+1.4,9.6+1.4$ and $8.4+1.4)$ when compared with the control $(11.2 \pm 0.4)$. The reduction could also be observed in the number of pups per litter $(8.1+1.3,9.0+1.5,5.1+1.7$ and 10.3 $\pm 0.6)$, and in the weigth of pups at birth $(2.1 \mathrm{~g} \pm 0.5,2.9 \mathrm{~g} \pm 0.4$, $\overline{1} .9 \mathrm{~g} \pm 0.1$ and $3.3 \mathrm{~g} \pm 0.1)$. We didn't observe any difference betwen $\mathrm{NvH}_{2} \mathrm{O}$, NvmeOH and control group. Macroscopicaly abnormalities were observed in some pups of Nvhex and Nvbut groups $(0.1 \%$ and $0.2 \%$ respectively). The results of the skeleton avaliation showed $33.3 \%, 45 \%, 9.8 \%$ e $11.1 \%$ of abnormalities for $\mathrm{NvH}_{2} \mathrm{O}$, NvmeOH, Nvbut and control group respectively. The most frequently observed abnormalities were found in the skull, and were: incomplete ossification of supraocciopital, interparietal, parietal, frontal and temporal.

Key words: calcinogenic plants, Nierembergia veitchii, vitamin $D_{3}$, infertility, teratogenic effects.

\section{INTRODUÇÃO}

A redução da fertilidade de rebanhos mantidos em pastagens contaminadas por plantas calcinogênicas tem sido objeto de observação de alguns autores (DIRKSEN et al., 1973; DÄMMRICH et al., 1975; DÖBEREINER et al., 1975; MELLO et al., 1995). Os estudos da toxicidade sistêmica e reprodutiva da administração, de pelo menos uma das duas plantas calcinogênicas prevalentes em nosso meio, têm demonstrado nítidos

\footnotetext{
${ }^{1}$ Professor Adjunto, Departamento de Farmacologia, Instituto de Ciências Básicas da Saúde, Universidade Federal do Rio Grande do Sul (UFRGS). Rua Sarmento Leite 500, Porto Alegre, RS, 90050-170. E-mail: jmello@ vortex.ufrgs.br. Autor para correspondência.

${ }^{2}$ Professor, Chemisches Institut - Tierärztliche Hochschule Hannover - Alemanha.

${ }^{3}$ Mestrando do Programa de Pós-graduação em Ciências Veterinárias, UFRGS.

${ }^{4}$ Bolsista de Iniciação Científica - PIBIC/CNPq
} 
efeitos de alguns extratos das plantas na fertilidade de ratos (MELLO et al., 1995). Essa redução da fertilidade pode ser observada quando machos e fêmeas são tratados antes da gestação com extrato aquoso de Solanum malacoxylon, ou quando fêmeas são tratadas durante toda a gestação (MELLO et al., 1996). Para a Nierembergia veitchii, os efeitos sobre a reprodução não são tão evidentes, embora tenham sido descritos redução da fertilidade e aparecimento de alterações morfológicas nos filhotes das ratas tratadas com extrato aquoso da planta (MELLO et al., 1997; BASTOS et al., 1998). O presente trabalho tem por objetivo investigar os efeitos da administração oral a ratas gestantes de quatro extratos da planta calcinogênica Nierembergia veitchii, avaliando parâmetros reprodutivos e os efeitos teratogênicos sobre as ninhadas.

\section{MATERIAL E MÉTODOS}

Nierembergia veitchii foi colhida no verão de 1995 na região de Pinheiro Machado, Rio Grande do Sul. A amostra foi identificada pelo Departamento de Botânica do Instituto de Biociências da UFRGS. Os extratos foram obtidos de 500g de planta seca e moída, de forma seqüencial em relação ao solvente: água $\left(\mathrm{NvH}_{2} \mathrm{O}\right)$, metanol $(\mathrm{NvmeOH})$, butanol (Nvbut) e hexano (Nvhex), concentrados em rotavapor e armazenados sob refrigeração até a administração, quando foram suspensos em água e/ou água + tween 80 e administrados por sonda gástrica aos animais.

Foram utilizados ratos e ratas albinos Wistar, com idade variando entre 120 e 150 dias, criados e mantidos no Biotério do Departamento de Farmacologia do Instituto de Ciências Básicas da Saúde da UFRGS, com período de luminosidade claro/escuro de 12 horas, temperatura de $21^{\circ} \mathrm{C} \pm$ $2^{\circ} \mathrm{C}$, alimentados com ração comercial e água $\boldsymbol{a d}$ libitum, acasalados por 12 horas (das 19 às 7 horas) na proporção de 1 macho para cada 3 fêmeas. Diariamente, foi realizado exame de esfregaço vaginal para constatação de cópula, pela presença de tampão e/ou espermatozóides. Este foi considerado o primeiro dia de gestação, e as fêmeas foram então individualizadas em gaiolas e passaram a receber por sonda gástrica, extrato aquoso $\left(\mathrm{NvH}_{2} \mathrm{O}\right)(\mathrm{n}=8)$, metanólico $(\mathrm{NvmeOH})(\mathrm{n}=8)$, butanólico (Nvbut) $(\mathrm{n}=8)$ e hexano (Nvhex) $(\mathrm{n}=7)$ de Nierembergia veitchii, vitamina $\mathrm{D}_{3}\left(2,5 \mathrm{mg} \cdot \mathrm{kg}^{-1}\right.$ por dia $)(\mathrm{n}=7)$ e solução fisiológica - grupo controle $(\mathrm{SF})(\mathrm{n}=6)$ em volume idêntico aos outros grupos. As doses de extrato utilizado correspondiam a $23,8 \mathrm{~g}$ de planta seca extraída por $\mathrm{kg}$, por dia. As fêmeas receberam o tratamento do $1^{\underline{0}}$ ao $21^{\underline{0}}$ dia de gestação, quando foram sacrificadas por secção cervical, para avalia- ção dos filhotes. Os parâmetros avaliados foram: peso diário das fêmeas, número de fêmeas copuladas, número de fêmeas gestantes, número de fêmeas paridas, número de implantes uterinos, número de filhotes por ninhada, número de reabsorções, peso dos filhotes ao nascerem, ocorrência de alterações morfológicas macroscópicas nos filhotes.

Após o sacrifício das fêmeas, o abdome foi aberto e o útero removido e pesado. $\mathrm{O}$ útero foi aberto e procedeu-se a contagem de filhotes e reabsorções, seguido de secagem, identificação individual, detecção de anomalias morfológicas macroscópicas e pesagem dos fetos. Os fetos das fêmeas dos grupos $\mathrm{NvH}_{2} \mathrm{O}$, NvmeOH, Nvbut e $\mathrm{SF}$, devidamente marcados, foram agrupados por ninhada e fixados com formol 5\%, por pelo menos 14 dias, e submetidos ao método de coloração óssea pela alizarina, segundo DAWSON (1926). Os fetos corados pela alizarina tiveram suas estruturas ósseas avaliadas individualmente em lupa, com ampliação de 10 vezes, e os resultados foram anotados por grupo. Foram avaliados: conformação, tamanho, relação com os demais ossos e grau de ossificação, conforme CHAHOUD (1996).

Os dados quantitativos, obtidos das médias dos parâmetros avaliados, foram tratados estatisticamente através da análise da variância e as taxas e percentuais foram comparados mediante o teste quiquadrado. Adotou-se como estatisticamente significativo o nível de 5\% (VIEIRA, 1981).

\section{RESULTADOS}

A figura 1 mostra o desenvolvimento ponderal das fêmeas tratadas com os 4 extratos de Nierembergia veitchii desde o dia da cópula até o vigésimo dia de gestação. Embora sejam observadas curvas ascendentes com relação à massa corporal das gestantes, os grupos tratados com Nvhex e Nvbut apresentaram menor ganho de peso, se avaliarmos a diferença do $1^{\circ}$ ao $20^{\circ}$ dia de tratamento. Isso também foi observado com o grupo tratado com vitamina $\mathrm{D}_{3}$. $\mathrm{O}$ diferencial em cada um dos grupos foi: $\mathrm{NvH}_{2} \mathrm{O}=91,2 \mathrm{~g}$; $\mathrm{NvmeOH}=94,8 \mathrm{~g}$; Nvbut = $72,7 \mathrm{~g}$; Nvhex $=53,6$; vitamina $\mathrm{D}_{3}=43,3$ e solução fisiológica $(\mathrm{SF})=98,0 \mathrm{~g}$. Os grupos tratados com $\mathrm{NvH}_{2} \mathrm{O}$ e $\mathrm{NvmeOH}$ apresentaram desenvolvimento ponderal semelhante ao observado no grupo controle (SF).

A tabela 1 resume os resultados referentes aos parâmetros reprodutivos obtidos após a administração oral dos extratos de Nierembergia veitchii $\left(\mathrm{NvH}_{2} \mathrm{O}, \mathrm{NvmeOH}, \mathrm{Nvbut}, \mathrm{Nvhex}\right)$ vitamina $\mathrm{D}_{3}$ e solução fisiológica durante toda a gestação. Os resultados mostram redução no número de implantes uterinos nos grupos tratados com Nvbut, Nvhex e vitamina $D_{3}$. A redução mostrou diferença estatisti- 


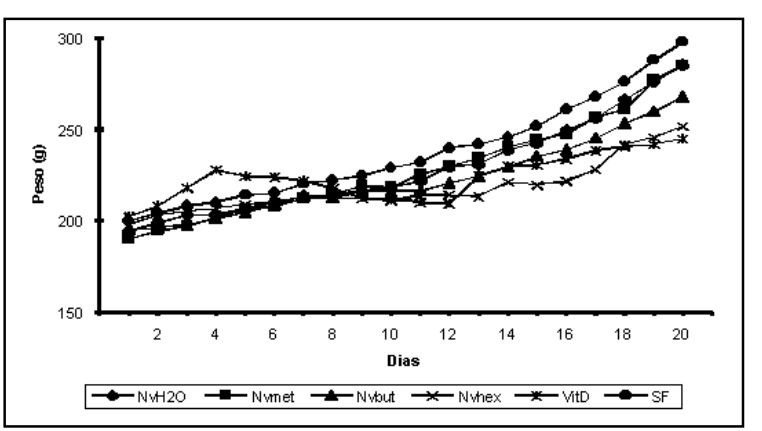

Figura 1 - Desenvolvimento ponderal de ratas tratadas por via oral com extratos de Nierembergia veitchii vitamina D3 e solução fisiológica durante toda a gestação

camente significativa somente nos dois últimos grupos, quando os dados foram comparados com o controle (SF). A redução do número de implantes uterinos repercutiu também no número de filhotes por ninhada. Embora Nvbut, Nvhex e vitamina $\mathrm{D}_{3}$ tenham apresentado ninhadas menores em relação ao controle, e aos grupos tratados com $\mathrm{NvH}_{2} \mathrm{O}$ e $\mathrm{NvmeOH}$, somente o grupo tratado com vitamina $\mathrm{D}_{3}$ acusou diferença estatisticamente significativa. No caso do número médio de reabsorções, novamente o grupo de fêmeas tratadas com vitamina $\mathrm{D}_{3}$ mostrou valores elevados e, significativamente, diferentes do controle. O grupo tratado com Nvhex mostrou um número de reabsorções embrionárias superior aos demais grupos $\left(\mathrm{NvH}_{2} \mathrm{O}, \mathrm{NvmeOH}\right.$, Nvbut, e solução fisiológica), porém os resultados não atingiram diferença estatisticamente significativa. Com relação ao peso médio dos filhotes ao nascerem, destacam-se novamente as reduções observadas nos grupos Nvbut, Nvhex e vitamina $D_{3}$, com significância estatística para os dois últimos. Nos grupos tratados com $\mathrm{NvH}_{2} \mathrm{O}$, NvmeOH, não houve diferença com relação ao grupo tratado com solução fisiológica. A taxa de natalidade dos grupos se manteve semelhante a do grupo controle, exceto para os grupos Nvhex e vitamina $\mathrm{D}_{3}$, nos quais foram observados $88 \mathrm{e}$ $61 \%$, respectivamente.

Nos grupos tratados com Nvbut e Nvhex, foram observados filhotes com anomalias morfológicas macroscópicas (Figura 2). Constavam de fetos menores, nos quais era difícil reconhecer e diferenciar estruturas ósseas da face, do tórax e dos membros. As alterações representaram taxas de anomalias de $0,2 \%$ para o grupo Nvbut e $0,1 \%$ para o grupo Nvhex.

Os fetos das fêmeas dos grupos $\mathrm{NvH}_{2} \mathrm{O}$, NvmeOH, Nvbut e SF, corados pela alizarina, mostraram, para os dois primeiros, elevadas taxas de anomalias, especialmente em ossos do crânio. Em especial, destacam-se ossificação incompleta, com deficiência de mineralização, de supraoccipital, interparietais, parietais, frontal e temporal (Tabela 2).

\section{RESULTADOS E DISCUSSÃO}

Os efeitos de plantas calcinogênicas sobre a reprodução de animais domésticos têm despertado crescente interesse (DIRKSEN et al., 1973; BARROS et al., 1992; MELLO et al., 1995; MELLO \& HABERMEHL, 1998). A deterioração do estado geral, indicando toxicidade sistêmica, tanto de machos quanto de fêmeas, parece explicar em parte, a infertilidade e as dificuldades de concepção apontadas (MELLO $\boldsymbol{e t} \boldsymbol{a l}$., 1995). Nessas condições, os sinais de dificuldade de locomoção, permanência por longos períodos deitados, diminuição do consumo de alimentos, perda de massa corporal, engrossamento de articulações, cifose, dificuldades respiratórias e arritmias cardíacas, observados em bovinos, ovinos, eqüinos, coelhos e ratos em condições experimentais e/ou de campo (DIRKSEN $\boldsymbol{e t} \boldsymbol{a l}$., 1973; TOKARNIA \& DÖBEREINER, 1974;

Tabela 1 - Parâmetros reprodutivos obtidos de ratas tratadas por via oral com extratos de Nierembergia veitchii $\left(\mathrm{NvH}_{2} \mathrm{O}, \mathrm{NvmeOH}\right.$, Nvbut, Nvhex) vitamina $\mathrm{D}_{3}$ e solução fisiológica durante toda a gestação.

\begin{tabular}{|c|c|c|c|c|c|c|c|c|c|c|}
\hline \multirow{2}{*}{$\begin{array}{l}\text { Parâmetro } \\
\text { Grupo }\end{array}$} & \multirow[t]{2}{*}{$\mathrm{n}$} & \multicolumn{2}{|c|}{ Implantes } & \multicolumn{2}{|c|}{ Filhotes } & \multicolumn{2}{|c|}{ Reabsorções } & \multirow{2}{*}{$\begin{array}{c}\mathrm{MC}^{\mathrm{b}}(\mathrm{g}) \\
\mathrm{x} \pm \mathrm{s}\end{array}$} & \multirow{2}{*}{$\begin{array}{l}\mathrm{N}^{\mathrm{c}} \\
(\%)\end{array}$} & \multirow{2}{*}{$\begin{array}{l}\mathrm{A}^{\mathrm{d}} \\
(\%)\end{array}$} \\
\hline & & $\mathrm{t}^{\mathrm{a}}$ & $\mathrm{x} \pm \mathrm{s}$ & $\mathrm{t}^{\mathrm{a}}$ & $\mathrm{x} \pm \mathrm{s}$ & $\mathrm{t}^{\mathrm{a}}$ & $\mathrm{x} \pm \mathrm{s}$ & & & \\
\hline $\mathrm{NvH}_{2} \mathrm{O}$ & 8 & 89 & $11,1 \pm 1,0$ & 86 & $10,7 \pm 1,2$ & 3 & $0,4 \pm 0,1$ & $3,0 \pm 0,3$ & 97 & 0 \\
\hline $\mathrm{NvmeOH}$ & 8 & 85 & $10,6 \pm 1,4$ & 81 & $10,1 \pm 1,0$ & 4 & $0,5 \pm 0,2$ & $3,3 \pm 0,2$ & 95 & 0 \\
\hline Nvbut & 8 & 77 & $9,6 \pm 1,4$ & 72 & $9,0 \pm 1,5$ & 5 & $0,6 \pm 0,3$ & $2,9 \pm 0,4$ & 94 & 0,2 \\
\hline Nvhex & 7 & 65 & $9,3 \pm 1,4^{*}$ & 57 & $8,1 \pm 1,3$ & 8 & $1,1 \pm 0,6$ & $2,1 \pm 0,5^{*}$ & 88 & 0,1 \\
\hline Vitamina $\mathrm{D}_{3}$ & 7 & 59 & $8,4 \pm 1,4 *$ & 36 & $5,1 \pm 1,7 *$ & 23 & $3,3 \pm 0,8 *$ & $1,9 \pm 0,1 *$ & 61 & - \\
\hline SF & 6 & 67 & $11,2 \pm 0,4$ & 62 & $10,3 \pm 0,6$ & 5 & $0,8 \pm 0,3$ & $3,3 \pm 0,1$ & 93 & 0 \\
\hline
\end{tabular}

${ }^{\mathrm{a}} \mathrm{t}$ - total; ${ }^{\mathrm{b}} \mathrm{MC}$ - massa corporal média dos filhotes; ${ }^{\mathrm{c}} \mathrm{N}$ - taxa de natalidade (número de filhotes vivos / número total de filhotes X 100); ${ }^{\mathrm{d}} \mathrm{A}$ - taxa de anomalias macroscópicas (número de filhotes com deformações macroscópicas externas / número total de filhotes X 100).

* Média diferente do respectivo controle, $\mathrm{p}<0,05$. 


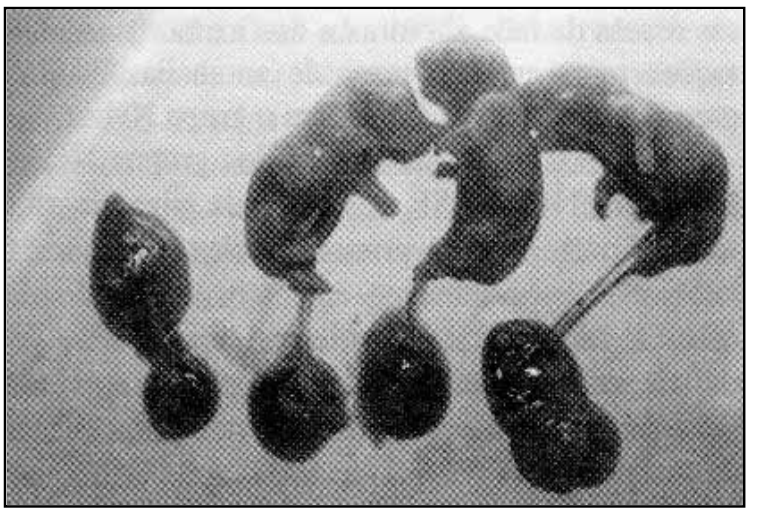

Figura 2 - Ninhada de fêmea tratada com Nvhex durante toda a gestação. Observa-se feto com tamanho reduzido em relação aos demais, com anomalias morfológicas em todo o corpo, dificultando identificação de estruturas.

WASSERMAN, 1978; RIET CORREA et al., 1987; BARROS et al., 1970; BARROS et al., 1992; MELLO et al., 1995), são predisponentes e, em alguns casos, determinantes da redução da fertilidade. Se para o Solanum malacoxylon esses sintomas e suas consequiências parecem evidentes (MELLO et al., 1995), para a Nierembergia veitchii, os efeitos sobre a reprodução são mais controversos. MELLO et al. (1996) relatam que o extrato aquoso de Nierembergia veitchii, mesmo sem produzir toxicidade materna (sistêmica) nítida, é capaz de interferir nos parâmetros reprodutivos de ratas. As evidências de que a presença de substâncias com atividade semelhante à da vitamina $\mathrm{D}_{3}$ na planta não fossem exclusivamente hidrossolúveis (MELLO, 1991; MELLO \& HABERMEHL, 1991; MELLO \& HABERMEHL, 1992; MELLO \& HABERMEHL, 1995; MELLO et al., 1995; MELLO \& HABERMEHL, 1998) levaram a investigação de extratos capazes de conter substâncias de solubilidade diferente. No presente experimento, os extratos Nvbut e Nvhex interferiram nos parâmetros reprodutivos investigados. Embora no caso do grupo Nvhex, possa-se suspeitar de toxicidade sistêmica, pela redução do desenvolvimento ponderal durante a gestação, no caso do grupo tratado com Nvbut isso não foi tão evidente. Apesar disso, alguns parâmetros reprodutivos foram adversamente afetados, aparentemente sem efeitos de toxicidade sistêmica. Com os extratos $\mathrm{NvH}_{2} \mathrm{O}$ e $\mathrm{NvmeOH}$, capazes de separar substâncias hidrossolúveis, os resultados não diferiram dos de fêmeas tratadas com solução fisiológica (controle). Isso talvez se justifique pela dose de extrato utilizada e pela presença de pequenas concentrações de princípio ativo hidrossolúvel na planta (MELLO, 1991; RIET CORREA et al., 1993). Em experimentos de campo, BARROS et al. (1992) acompanharam quatro ovelhas com sintomas de calcinose por Nierembergia veitchii quanto à evolução da sintomatologia. Observaram que a fertilidade não era afetada, pois duas fêmeas adultas conceberam durante a fase ativa da doença, parindo cordeiros saudáveis a termo, voltando a fazê-lo na monta seguinte. Concluíram que, apesar das evidências encontradas, o pequeno número de casos não permitia uma conclusão com relação aos efeitos sobre a fertilidade.

Anomalias morfológicas fetais já haviam sido descritas para o extrato aquoso de Nierembergia veitchii, bem como para a vitamina $\mathrm{D}_{3}$ (MELLO et al., 1997; BASTOS et al., 1998). No presente experimento, as anomalias macroscópicas foram observadas nos filhotes de fêmeas tratadas com Nvbut e Nvhex. No caso dos extratos $\mathrm{NvH}_{2} \mathrm{O}$ e $\mathrm{NvmeOH}$, em que pese a ausência de efeitos sobre os parâmetros reprodutivos avaliados, houve elevado percentual de anomalias ósseas nos fetos, confirmando sua ação sobre a formação óssea dos mesmos. Mais uma vez se confirmam as suspeitas da presença de substâncias com atividade semelhante à da vitamina $\mathrm{D}_{3}$, com diferentes características de solubilidade.

Toxicidade pré-implantação, se presente, deve ser em grau reduzido, uma vez que a diferença entre o número de implantes uterinos e número de filhotes não diferiu entre os grupos. Ao contrário, a constatação de anomalias fetais aponta no sentido da toxicidade pós-implantação para os extratos testados. Segundo LEMÔNICA et al. (1996), o período de pré-implantação, que ocorre na rata do primeiro ao sexto dia de gestação, é considerado um período de "tudo ou nada", em que a exposição materna a substâncias químicas pode causar tanto a morte do embrião, quanto o desenvolvimento normal de fetos. O período de organogênese ocorre do sexto ao dé-

Tabela 2 - Anomalias ósseas em fetos de ratas tratadas por via oral com extratos de Nierembergia veitchii $\left(\mathrm{NvH}_{2} \mathrm{O}\right.$, NvmeOH, Nvbut e solução fisiológica) durante toda a gestação.

\begin{tabular}{|c|c|c|c|c|}
\hline Anomalia & $\mathrm{NvH}_{2} \mathrm{O}$ & $\mathrm{NvmeOH}$ & Nvbut & SF \\
\hline Número de fetos & 63 & 60 & 51 & 54 \\
\hline${ }^{\mathrm{a}}$ Interparietais & $20,6 \% *$ & $25,0 *$ & zero & $1,9 \%$ \\
\hline${ }^{a}$ Parietais & $28,6 \% *$ & $36,6 \% *$ & $5,9 \%$ & $7,4 \%$ \\
\hline${ }^{\mathrm{a}}$ Supraoccipital & $7,9 \% *$ & $11,7 \% *$ & zero & $1,8 \%$ \\
\hline${ }^{\mathrm{a}}$ Zigomático & zero & $3,3 \%$ & zero & $1,8 \%$ \\
\hline${ }^{\mathrm{a}}$ Frontal + Temporal & $7,9 \% *$ & $20,0 \% *$ & zero & $1,8 \%$ \\
\hline${ }^{\mathrm{a}}$ Esterno & $1,6 \%$ & $3,3 \%$ & zero & zero \\
\hline${ }^{\mathrm{b}}$ Costelas & zero & $1,7 \%$ & zero & zero \\
\hline
\end{tabular}

${ }^{\mathrm{a}}$ Ossificação incompleta; ${ }^{\mathrm{b}}$ Arqueamento

* Valor diferente do respectivo controle. $\mathrm{v}<0.05$. 
cimo quinto dia, ocorrendo aí as alterações morfológicas.

A constatação da presença de receptores para vitamina $\mathrm{D}_{3}$ em tecidos e órgãos ligados à função reprodutiva, como ovários, útero, placenta, glândula mamária (DOKOH et al., 1983), células de Sertoli e túbulos seminíferos (MERKE et al., 1985), apontam no sentido da participação da substância e seus metabólitos na função reprodutiva. Portanto, a constatação da presença de substâncias com atividade semelhante à da vitamina $\mathrm{D}_{3}$ na planta calcinogênica Nierembergia veitchii parece justificar a toxicidade reprodutiva observada.

\section{REFERÊNCIAS BIBLIOGRÁFICAS}

BARROS, S.S., POHLENZ, J., SANTIAGO, C. Zur Kalzinose beim Schaf. Dtsch Tierärztl Wschr, v.77, p.346-349, 1970.

BARROS, S.S., DRIEMEIER, D., SANTOS, M.N., et al. Evolução clínica e reversibilidade das lesões da calcinose enzoótica dos ovinos induzida por Nierembergia veitchii. Pesq Vet Bras, v.12, n.1/2, p.5-10, 1992.

BASTOS, F.C., MELlO, J.R.B., LANGELOH, A., $\boldsymbol{e t}$ al. Ocorrência de anomalias ósseas em fetos de ratas tratadas com extratos de Nierembergia veitchii Solanaceae. In: XIII REUNIÃO ANUAL DA FEDERAÇÃO DE SOCIEDADES DE BIOLOGIA EXPERIMENTAL, 1998, Caxambu, MG Anais... São Paulo : FESBE, 1998. 494p. p.133.

CHAHOUD, I. Atlas of External and Skeletal Anormalies in Rats. Berlin : Institute of Toxicology and Embryopharmacology FU, Leipzig : Pr \& C GmbH, 1996. CD Room.

DÄMMRICH, K., DÖBEREINER, J., DONE, S.H., et al. Skelettveränderungen nach Vergiftungen mit Solanum malacoxylon bei Rindern. Zentralbl Veternärmed A, v.22, p.313-329, 1975.

DAWSON, A.B. A note on the staining of the skeleton of cleared specimens with Alizarin Red S. Stain Technology, v.1, p.123-124, 1926.

DIRKSEN, G., PLANK, P., HÄNICHEN, T., et al. Die enzootische Kalzinose - eine neue Weidekrankheit des Rindes. Tierzüchter, v.25, p.150-153, 1973

DÖBEREINER, J., TOKARNIA, C.H., COSTA, C.B.D., et $\boldsymbol{a l}$. "Espichamento", intoxicação de bovinos por Solanum malacoxylon no Pantanal do Mato Grosso. Pesq Agropec Bras, Ser Vet, v.6, p.91-117, 1975.

DOKOH, S., DONALDSON, C.A., MARION, S.L., et al. The ovary: a target organ for 1,25-dihydroxyvitamin $D_{3}$. Endocrinology, v.112, p.200-206, 1983.

LEMÔNICA, I.P., DAMASCENO, D.C., DI-STASI, L.C. Study of the embryotoxic effects of an extract of rosemary (Rosamarinus officinalis L.). Brazilian Journal of Medical and Biological Research, v.29, n.2, p.223-227, 1996.

MELLO, J.R.B. Untersuchungen der Auswirkungen von kalzinogenen Pflanzen auf die elemente $\mathrm{Ca}, \mathrm{P}$ und alkalische phosphatase bei Hühnerküken. Hannover, 1991. 196p. Diss. (Doctor Medicinae Veterinariae) - Curso de Pósgraduação em Medicina Veterinária, Tierärztliche Hochschule
Hannover, 1991

MELLO, J.R.B., HABERMEHL, G. Einfluss ultravioletter Strahlen und klimatischer Bedingungen (Sonneneinstrahlung und Temperatur) auf die kalzinogene Aktivität von Trisetum flavescens und Nierembergia veitchii. Dtsch Tierärztl Wschr, v.98, p.441-476, 1991

MELLO, J.R.B., HABERMEHL, G. Kalzinogene pflanzen und der inkubationseffekt durch pansensaft. Dtsch Tierärztl Wschr, v.99, p.353-392, 1992.

MELLO, J.R.B., HABERMEHL, G. Substâncias com atividade similar à vitamina $\mathrm{D}_{3}$ em quatro plantas calcinogênicas. Pesq Vet Bras v.15, n.2/3, p.73-78, 1995.

MELLO, J.R.B., ALMEIDA, C.R.C., ZANDER, R., et al. O envolvimento de substâncias com atividade vitamina D na etiologia da infertilidade produzida pela planta calcinogênica Solanum malacoxylon Solanaceae. Arq Fac Vet UFRGS, v.22, p.9-19, 1995

MELlO, J.R.B., LANGELOH, A ., ALMEIDA, C., et al. Avaliação da fertilidade de ratas tratadas com Solanum malacoxylon Solanaceae e Nierembergia veitchii Solanaceae. In: REUNIÃO ANUAL DA FEDERAÇÃO DE SOCIEDADES DE BIOLOGIA EXPERIMENTAL, 11, 1996, Caxambu, MG. Anais... São Paulo : FESBE, 1996. 340p. p.399-340.

MELlO, J.R.B., LANGELOH, A ., ALMEIDA, C., et al. Estudo de extratos de Nierembergia veitchii Solanaceae sobre a fertilidade de ratas. In: REUNIÃO ANUAL DA FEDERAÇÃO DE SOCIEDADES DE BIOLOGIA EXPERIMENTAL, 12, 1997, Caxambu, MG. Anais... São Paulo : FESBE, 1997. 431p. p.132.

MELLO, J.R.B., HABERMEHL, G. Untersuchungen der Auswirkungen von kalzinogenen Pflanzen - Qualitative und quantitative Bewertung. Dtsch Tierärztl Wschr, v.105, p.2529, 1998 .

MERKE, J., HÜGEL, U., RITZ, E. Nuclear testicular 1,25dihydroxyvitamin $\mathrm{D}_{3}$ receptors in Sertoli cells and seminiferous tubules of adult rodents. Biochemical and Biophysical Research Communications, v.127, p.303-309, 1985

RIET CORREA, F., SCHILD, A.L., MÉNDEZ, M.C., et al. Enzootic calcinosis in sheep caused by the ingestion of Nierembergia veitchii (Solanaceae). Pesq Vet Bras, v.7, n.3, p.85-95, 1987

RIET CORREA, F., MÉNDEZ, M.C., SCHILD, A.L., et al. Enzootic calcinosis in sheep. Experimental reproduction with Nierembergia veitchii (Solanaceae). Pesq Vet Bras v.13, n.1/2, p.21-24, 1993.

TOKARNIA, C.H., DÖBEREINER, J. "Espichamento", intoxicação de bovinos por Solanum malacoxylon, no Pantanal do Mato Grosso. II. Estudos complementares. Pesq Vet Bras v.9, p.53-62, 1974.

VIEIRA, S. Introdução a bioestatística. Rio de Janeiro: Campus, 1981. 294p.

WASSERMAN, R.H. The nature and mechanism of action of the calcinogenic principle of Solanum malacoxylon and Cestrum diurnum and comment on Trisetum flavescens. In: KELLER, R.F., KAMPEN, K.R., JAMES, L.F. Effects of poisonous plants on livestock. New York : Academic, 1978. p.545553 